\author{
EVS27 \\ Barcelona, Spain, November 17-20, 2013
}

\title{
Motor-Generator Control to Improve Shift Quality for a Dual Mode Power Split Transmission
}

\author{
Sungwha Hong ${ }^{1}$, Woulsun Choi ${ }^{1}$, Sunghyun Ahn ${ }^{1}$, Geontae Lee ${ }^{1}$, Hanho Son ${ }^{1}$, \\ Yongjoo $\mathrm{Kim}^{2}$ and Hyunsoo $\mathrm{Kim}^{1}$ \\ ${ }^{I}$ School of Mechanical Engineering,Sungkyunkwan University, Suwon-si, Republic of Korea, hskim@me.skku.ac.kr \\ ${ }^{2}$ Machinery Technology Group, Advanced R\&D Center, LS Mtron Ltd, Gunpo-si, Republic of Korea
}

\begin{abstract}
In this paper, a motor-generator (MG) control algorithm was proposed to improve the shift quality for a dual mode power split transmission (PST) hybrid electric vehicle (HEV). In order to analyze the shift characteristics during the mode shift, Bondgraph models for transient-state were constructed and state equations were derived. From the state equations, it was found that inertia torque of MG2 causes a transient torque during the mode shift. Based on the transient torque, a MG torque control algorithm which compensates the transient torque was developed to reduce the driveshaft torque variation. To evaluate the performance of the MG2 control algorithm, dynamic models of the dual mode PST HEV were obtained and an AMESim-MATLAB/Simulink based mode shift performance simulator was developed. It was found from the simulation results that the driveshaft torque variation was reduced by the proposed control algorithm which provides improved shift quality.
\end{abstract}

Keywords: dual mode PST, motor-generator, mode shift, motor torque control

\section{Introduction}

A power split type hybrid electric vehicle (HEV) transmits power through the power split transmission (PST) which consists of electrical path and mechanical path. The electrical path is composed of motor-generators (MG) meanwhile the mechanical path is composed of planetary gear sets.

The PST can be divided into input split, output split, compound split type depending on the location of power split [1]. When one of the PSTs is singly used, it is called 'single mode PST', and when two of above PSTs are combined, it is called 'dual mode PST'. Toyota Prius, Ford Hybrid Escape are single mode PST, which only use the input split. In this type, a power circulation which occurs at high speed has been pointed out as a main drawback [2].

To solve this problem, a dual mode PST, wherein the clutch and brake are used for the mode shift, has been investigated for ensuring high efficiency both at high and low speed [3,7]. The dual mode PST has an advantage that more efficient PST configuration can be selected according to the driving conditions [4]. However, a torque variation which may occur during the mode shift causes poor shift quality. Many researches have been performed on the gear shift for automatic transmission (AT), dual clutch transmission (DCT) $[5,6]$. However, few researches have been conducted for the dual mode PST.

In this study, a MG control algorithm to improve shift quality is proposed for a dual mode PST. Performance of the proposed control algorithm is 
evaluated using AMESim-MATLAB/Simulink simulator.

\section{Structure of dual mode power split transmission}

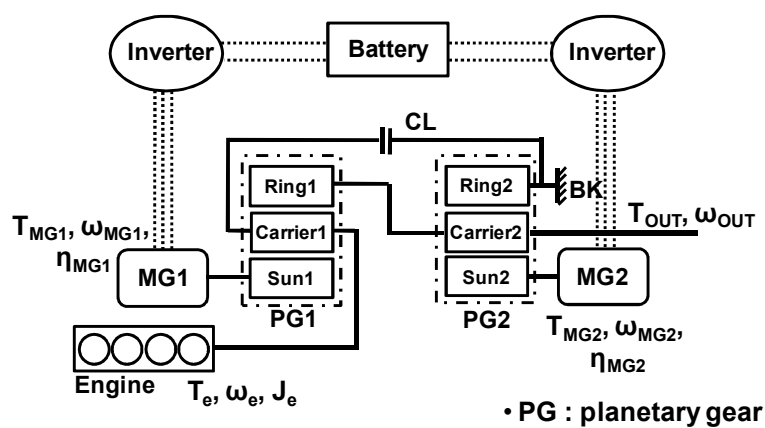

Figure1: Structure of dual mode PST

Table1: Friction elements operation of dual mode PST

\begin{tabular}{|l|c|c|c|}
\hline & $\begin{array}{c}\text { Engine } \\
\text { on }\end{array}$ & $\begin{array}{c}\text { Clutch } \\
\text { (CL) }\end{array}$ & $\begin{array}{c}\text { Brake } \\
\text { (BK) }\end{array}$ \\
\hline EV mode & & & $\mathrm{O}$ \\
\hline Input split mode & $\mathrm{O}$ & & $\mathrm{O}$ \\
\hline $\begin{array}{l}\text { Compound split } \\
\text { mode }\end{array}$ & $\mathrm{O}$ & $\mathrm{O}$ & \\
\hline
\end{tabular}

Fig. 1 shows the configuration of the investigated dual mode PST. The dual mode PST consists of an engine, two motor-generators (MG), two planetary gears (PG), a clutch (CL) and a brake (BK). The engine is connected to the carrier of PG1 (carrier1), and the MG1 is connected to the sun gear of PG1 (sun1). The MG2 is connected to the sun gear of PG2 (sun2), and the output shaft is connected to the carrier of PG2 (carrier2). The CL is located between carrier1 and ring2, and $\mathrm{BK}$ is attached to ring2. The mode shift between the input split and the compound split mode is performed according to the operation of these friction elements. Tablel shows the operation of friction elements at each mode. The input split mode is realized by engaging the BK while disengaging the $\mathrm{CL}$; and the compound split mode is realized by engaging the CL while disengaging the BK. The EV mode is implemented by turning off the engine at the input split mode and the vehicle is driven only by the MG2. The dual mode PST under study has four cases of mode shifts: 1)from EV to input split mode; 2)from input split to compound split mode; 3 )from compound split to input split mode, 4)from compound split to EV mode. In this study, the second case, 2)from input split to compound split mode, is investigated.

\section{Transient-state model for mode shift}

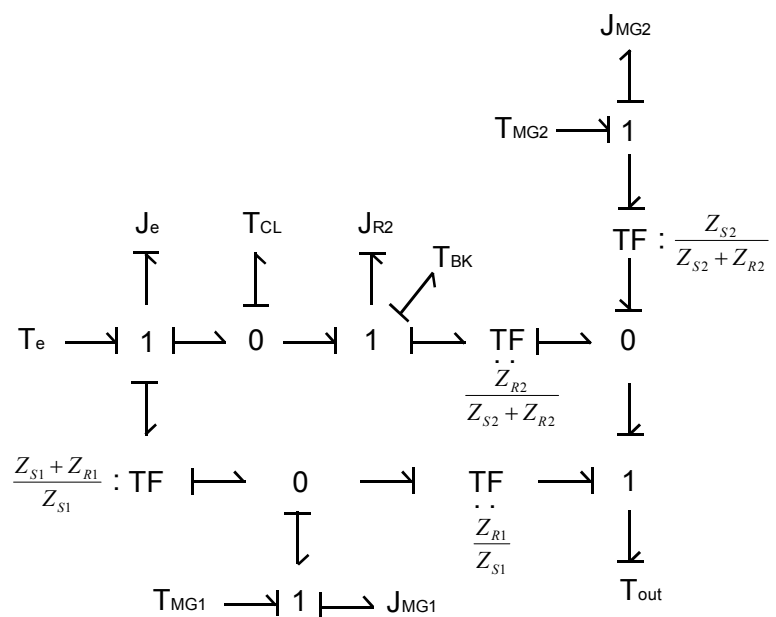

Figure2: Bondgraph model of transient-state (from the input split mode to the compound split mode)

In order to analyze the shift characteristics during mode shift, Bondgraph model for the transientstate is derived (Fig. 2). Since the Bondgraph model includes torque and inertia of the engine/MG1/MG2 and transfer torque of $\mathrm{CL} / \mathrm{BK}$, influence of each component can be analyzed. From Bondgraph model, the transient-state equations for the mode shift are obtained as follows:

$$
\begin{aligned}
& T_{O U T}=\frac{Z_{S 2}+Z_{R 2}}{Z_{S 2}} T_{M G 2}-\frac{Z_{R 1}}{Z_{S 1}} T_{M G 1} \\
& -\frac{Z_{R 2}+Z_{S 2}}{Z_{S 2}} J_{M G 2} \dot{\omega}_{M G 2}+\frac{Z_{R 1}}{Z_{S 1}} J_{M G 1} \dot{\omega}_{M G 1} \\
& J_{e} \dot{\omega}_{e}=T_{e}+\frac{Z_{S 1}+Z_{R 1}}{Z_{S 1}} T_{M G 1} \\
& -\frac{Z_{S 1}+Z_{R 1}}{Z_{S 1}} J_{M G 1} \dot{\omega}_{M G 1} \\
& J_{R 2} \dot{\omega}_{R 2}=-T_{C L}-T_{B K}-\frac{Z_{R 2}}{Z_{S 2}} T_{M G 2} \\
& +\frac{Z_{R 2}}{Z_{S 2}} J_{M G 2} \dot{\omega}_{M G 2} \\
& \dot{\omega}_{O U T}=\frac{Z_{R 2}}{Z_{S 2}+Z_{R 2}} \dot{\omega}_{R 2} \\
& +\frac{Z_{S 2}}{Z_{S 2}+Z_{R 2}} \dot{\omega}_{M G 2}
\end{aligned}
$$


$\dot{\omega}_{M G 1}=\frac{Z_{S 1}+Z_{R 1}}{Z_{S 1}} \dot{\omega}_{e}-\frac{Z_{R 1}}{Z_{S 1}} \dot{\omega}_{\text {OUT }}$

where $T_{\text {OUT }}$ is the transmission output shaft torque, $Z_{S}$ is the number of teeth of sun gear, $Z_{R}$ is the number of teeth of ring gear. Subscripts 1 and 2 indicate the first and second planetary gear, respectively. $T$ is the torque, $\omega$ is the rotational speed, and $J$ is the inertia.

Eq. (1) is the output shaft torque of the transientstate during mode shift. From Eq. (1), it is found that the output shaft torque of the transient-state is affected by the inertia torque of MG1/MG2 as well as the steady-state torque of MG1/MG2. As shown in Eq. (2), the engine speed variation $\dot{\omega}_{e}$ is influenced by the MG1 torque, $T_{M G 1}$ but independent from the vehicle speed, which means that the engine speed can be operated in high efficiency region by the MG1 torque control.

\section{MG control algorithm}

\subsection{MG1 control}

In the dual mode PST under study, MG1 is responsible for the optimal engine operation. Therefore, in the steady-state, the engine is controlled to follow the demanded engine speed, which is calculated based on the engine optimal operating line (OOL) $[7,8]$.

Eq. (5) is the speed equation of the MG1, engine and output shaft. Since the vehicle inertia is very large, the output shaft speed variation $\dot{\omega}_{\text {OUT }}$ is very small and can be ignored. The engine speed variation causes the MG1 speed variation. From Eq. (1), it is seen that the output shaft torque during the mode shift is affected by the inertia torque of MG1, hence, the MG1 speed variation may cause the output shaft torque variation. Thus, the engine speed during the mode shift needs to be controlled to follow the demanded engine speed.

\subsection{MG2 control}

In Eq. (1), the MG1 inertia torque $J_{M G 1} \dot{\omega}_{M G 1}$ can be ignored because the MG1 speed is hardly changed while the engine speed is maintained on the OOL as explained in 4.1. Then, the MG2 inertia torque $J_{M G 2} \dot{\omega}_{M G 2}$ plays the main role that causes transient torque of the output shaft during mode shift. In this study, a MG2 torque control is proposed to compensate the inertia torque of MG2 as follows:

The magnitude of the MG2 inertia torque is expressed as,

$T_{\text {OUT_inertia }}=-\frac{Z_{S 2}+Z_{R 2}}{Z_{S 2}} J_{M G 2} \dot{\omega}_{M G 2}$

Substituting $\dot{\omega}_{M G 2}$ in Eq. (3) to Eq. (6), $T_{\text {OUT_inertia }}$ can be expressed as follows:

$$
\begin{aligned}
& T_{O U T_{-} \text {inertia }}=-\frac{Z_{S 2}+Z_{R 2}}{Z_{R 2}} J_{R 2} \dot{\omega}_{R 2} \\
& -\frac{Z_{S 2}+Z_{R 2}}{Z_{S 2}} T_{M G 2}-\frac{Z_{S 2}+Z_{R 2}}{Z_{R 2}} T_{C L} \\
& -\frac{Z_{S 2}+Z_{R 2}}{Z_{R 2}} T_{B K}
\end{aligned}
$$

Since ring2 inertia $J_{R 2}$ is very small, inertia torque $J_{R 2} \dot{\omega}_{R 2}$ can be ignored. The magnitude of compensation torque, $T_{M G 2}$ in Eq. (7) that makes the output shaft inertia torque $T_{\text {OUT_inertia }}$ zero can be obtained as,

$$
\begin{aligned}
& T_{M G 2}=-\frac{Z_{S 2}+Z_{R 2}}{Z_{R 2}} T_{C L} \\
& -\frac{Z_{S 2}+Z_{R 2}}{Z_{R 2}} T_{B K}
\end{aligned}
$$

In Eq. (8), clutch torque $T_{C L}$ and brake torque $T_{B K}$ are determined by the clutch/brake pressure. The demanded MG2 torque during the mode shift is obtained by combining the MG2 torque for the steady-state $T_{M G 2} *$ and the MG2 compensation torque $T_{M G 2}$ as,

$T_{M G 2_{-} d m d}=T_{M G 2} *+T_{M G 2}$

where $T_{M G 2} *$ is the steady-state MG2 torque.

The demanded MG2 torque $T_{M G 2 \text { dmd }}$ is applied during the inertia phase of the mode shift, where the speed of ring gear $R 2$ increases from zero to the engine speed.

\section{Mode shift performance simulator for dual mode PST}

Fig. 3 shows the mode shift performance simulator for dual mode PST. The powertrain model is developed based on AMESim, and the controller is developed based on MATLAB/Simulink. The 
powertrain model consists of engine, torsion damper, MGs, planetary gear set and vehicle model. And the controller consists of driver model, engine control, torque calculation of MGs, mode selection and pressure control of $\mathrm{CL} / \mathrm{BK}$.

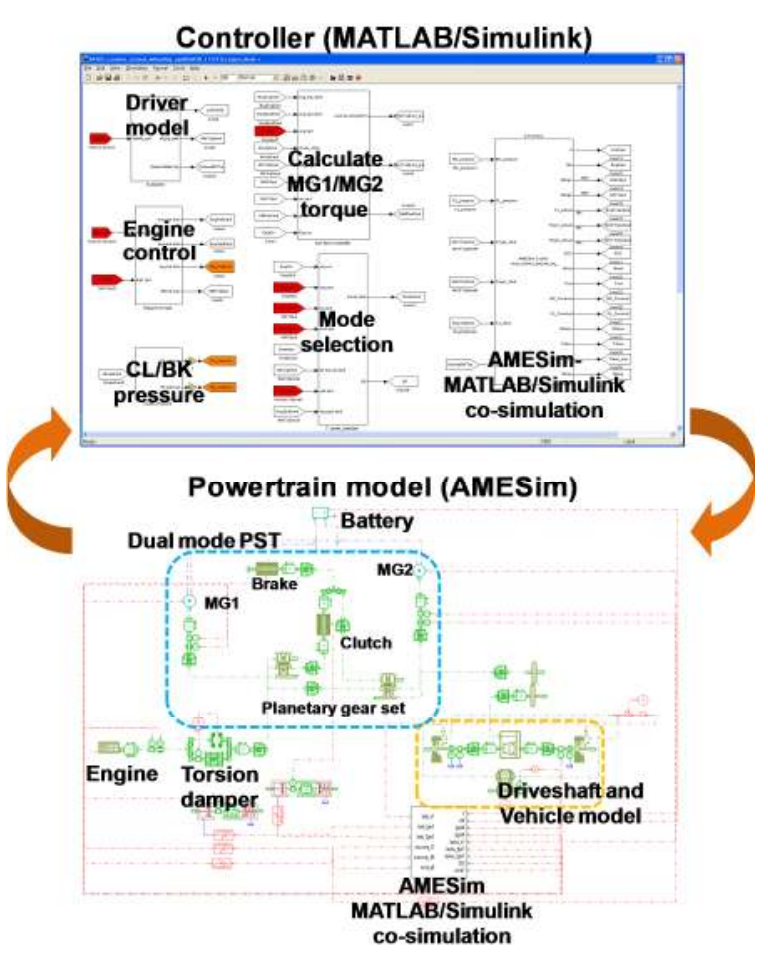

Figure3: Mode shift performance simulator

\section{Simulation results}

The performance of the proposed MG control algorithm is evaluated using the mode shift performance simulator. Driving profile in Fig. 4(a) is used for simulation. Fig. 4 shows the mode shift performance simulation results for the mode shift from the input split mode to the compound split mode.

When a mode shift signal is given at point $\mathrm{S}$ (a), BK pressure decreases and CL pressure increases (b). The MG1 torque is controlled to make the engine follow the demanded engine speed (c). The MG2 torque with control (c) shows a decrease in the inertia phase by the amount of compensation torque while the MG2 torque without control shows a demanded torque at a steady-state. During the transient-state, the MG1 speed is maintained almost constant while the MG2 speed is rapidly changed(d). The engine actual speed follows the demanded engine speed closely by the MG1 torque control(e). After the mode signal is activated at point $\mathrm{S}$, the actual demanded engine speed(e). It is because the MG1 torque is rapidly changed at point $\mathrm{S}(\mathrm{c})$.
The peak to peak driveshaft torque is reduced from $89 \mathrm{Nm}$ to $63.7 \mathrm{Nm}$ by $34.2 \%$ using the MG torque control (f).
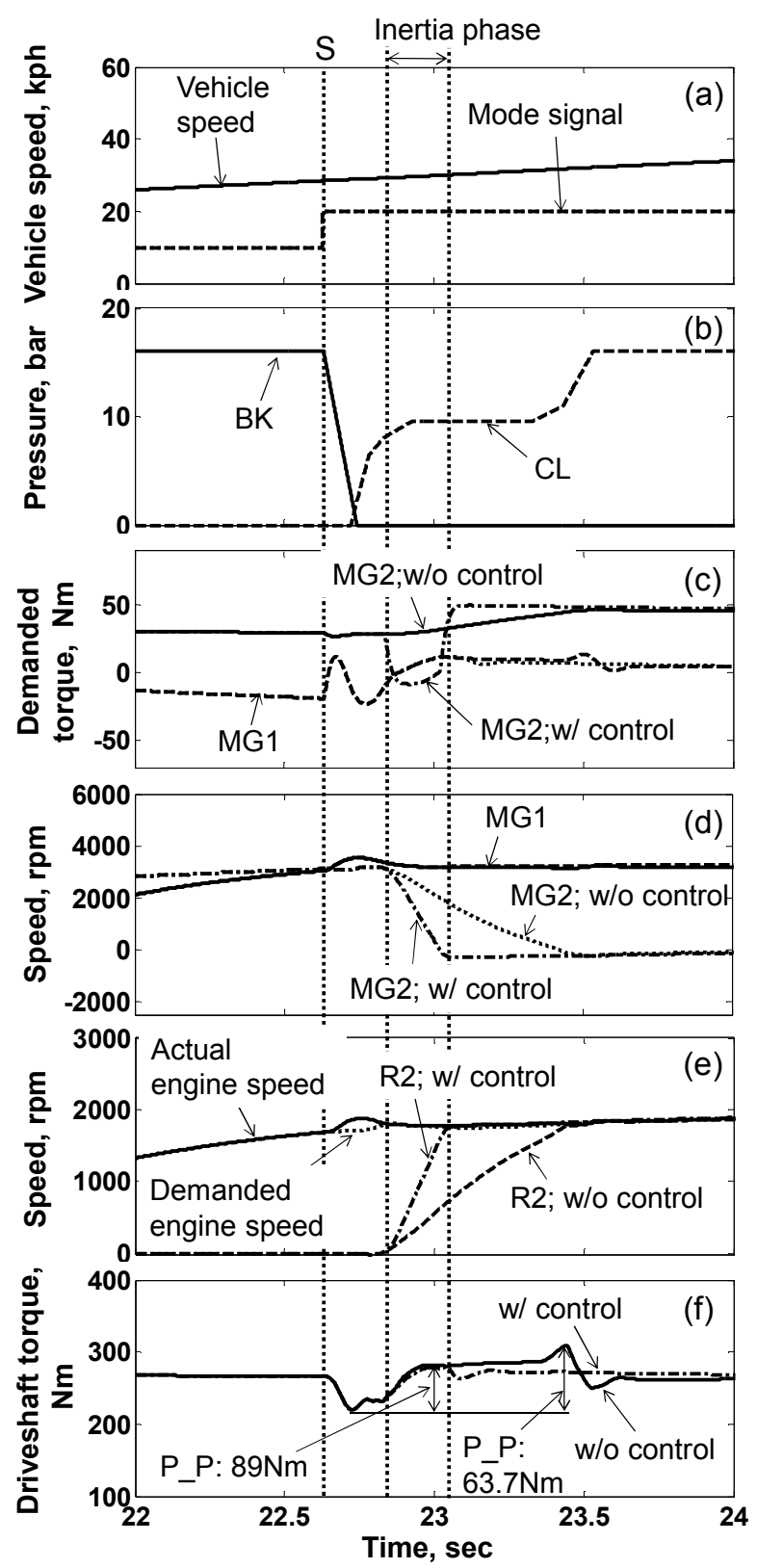

Figure4: Simulation results with MG control algorithm (from the input split mode to the compound split mode)

\section{Conclusions}

A MG control algorithm for dual mode PST was proposed, which controls the MG2 torque to reduce the driveshaft torque variation during the mode shift. To evaluate the shift performance of the dual mode PST, a shift performance simulator was developed. In order to analyze the shift characteristics during the mode shift, Bondgraph 
models for the transient-state were constructed and state equations were derived. From the state equations, it was found that the driveshaft torque variation was caused by the inertia torque of MG2. Based on the transient torque characteristics, a MG torque control algorithm which compensates the inertia torque was proposed and its performance was investigated using the simulator. The simulation results showed that the driveshaft torque variation was reduced by the proposed MG control algorithm, which provides the improved shift quality for dual mode PST.

\section{References}

[1] T. Yaegashi et. Al., Toyota Hybrid System : It's Concept and Technologies, FISTA98 F98TP095, 2004

[2] B. Conlon, Comparative Analysis of Single and Combined Hybrid Electrically Variable Transmission Operating Modes, SAE 200501-1162, 2005

[3] B. Mashadi and S. Emadi, Dual-Mode Power-Split Transmission for Hybrid Electric Vehicle, IEEE Transactions on Vehicular Technology, Vol. 59, No. 7, pp. 3223-3232, 2010

[4] N. Kim et. Al., Control Strategy for a DualMode Electromechanical, Infinitely Variable Transmission for Hybrid Electric Vehicles, Proceedings of the Institution of Mechanical Engineers, Part D;Journal of Automotive Engineering, Vol. 222, pp. 1587-1601, 2008

[5] K. Sanada et. Al., Design of a Robust Controller for Shift Control of an Automatic Transmission, Proceedings of the Institution of Mechanical Engineers, Part D; Journal of Automobile Engineering, Vol. 226, pp. 1577-1584, 2012

[6] M. Wu et. Al., Research on Optimal Control for Dry Dual-clutch Engagement during Launch, Proceedings of the Institution of Mechanical Engineers, Part D; Journal of Automobile Engineering, Vol. 224, pp. 749-763, 2010

[7] W. Choi et. Al., Development of a Control Algorithm to Reduce Torque Variation for the Dual Mode HEV During Mode Shift, Vehicle Power and Propulsion Conference 12339357, 2011

[8] J. Kim et. Al., Mode Control Strategy for a Two-Mode Hybrid Electric Vehicle using Electrically Variable Transmission(EVT) and Fixed-Gear Mode, IEEE Transaction on Vehicular Technology, Vol. 60, No. 3, 2011

\section{Authors}

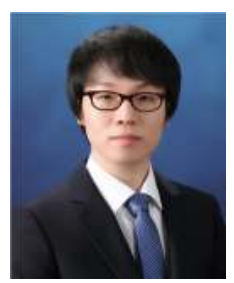

Sungwha Hong received a bachelor degree in mechanical engineering from Sungkyunkwan University, Korea, in 2011. He is currently working toward the Ph.D. degree with the School of Mechanical Engineering, Sungkyunkwan University. His research interests include the modeling, design of powertrain system and shift quality of hybrid electric vehicle, electric vehicle.

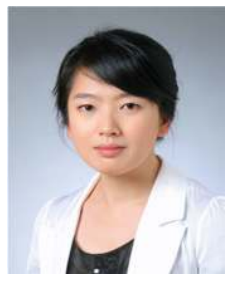

Woulsun Choi received her M.S degree in mechanical engineering from Sungkyunkwan University, Korea, in 2012. Since 2012, she has worked as research engineer of Hyundai Motor Company. Her research interests include the modeling, control, test of powertrain system for hybrid electric vehicle

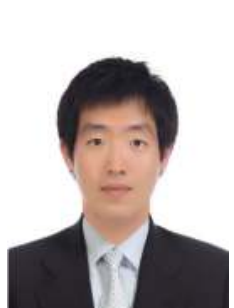

Sunghyun Ahn received a bachelor degree in mechanical engineering from Sungkyunkwan University, Korea, in 2012 where he has been working toward M.S degree. His research interests include the modeling and control of powertrain system electric vehicle and agricultural vehicle.

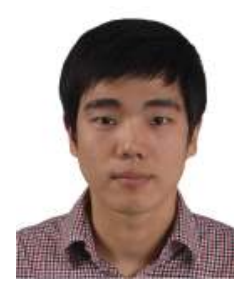

Geontae Lee received a bachelor degree in mechanical engineering from Sungkyunkwan University, Korea, in 2013 where he has been working toward M.S degree. His research interests include the NVH of the powertrain system for hybrid electric vehicle.

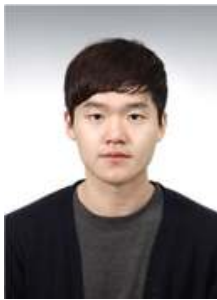

Hanho Son received a bachelor degree in mechanical engineering from Sungkyunkwan University, Korea, in 2013 where he has been working toward M.S degree. His research interests include the modeling, design, and control of powertrain system for hybrid electric vehicle and electric vehicle 


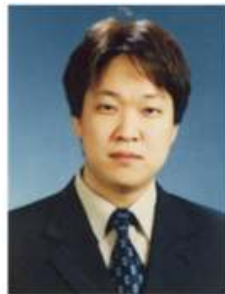

Yongjoo Kim received a Ph.D. degree in agricultural engineering from the Sungkyunkwan university at Suwon, Gyeonggi-do, Korea, in 2008. Since 2008, he has worked as a senior research engineer, project manager of advanced $R \& D$ center tractor division at LS Mtron Ltd

Hyunsoo Kim received a Ph.D. degree in mechanical engineering from the University of Texas at Austin, Texas, USA, in 1986. Since 1986, he has worked as a Professor, Chairman, and Dean of the College of Engineering at Sungkyunkwan University. His main research interests include Hybrid Electric Vehicle (HEV) transmission system design, regenerative braking, and optimal power-distribution algorithms for HEV and vehicle stability control for HEV and In-wheel Electric Vehicles. 